\title{
SEPARABLE SUBALGEBRAS OF A CLASS OF AZUMAYA ALGEBRAS
}

\author{
GEORGE SZETO \\ Department of Mathematics \\ Bradley University \\ Peoria, Illinois 61625, U.S.A. \\ (Received July 15, 1996 and in revised form April 14, 1997)
}

\begin{abstract}
Let $S$ be a ring with $1, C$ the center of $S, G$ a finite automorphism group of $S$ of order $n$ invertible in $S$, and $S^{G}$ the subnng of elements of $S$ fixed under each element in $G$. It is shown that the skew group ring $S * G$ is a $G^{\prime}$-Galois extension of $(S * G)^{G^{\prime}}$ that is a projective separable $C^{G}$-algebra where $\mathrm{G}^{\prime}$ is the inner automorphism group of $S * G$ induced by $G$ if and only if $S$ is a $G-G a l o i s$ extension of $S^{G}$ that is a projective separable $C^{G-a l g e b r a . ~ M o r e o v e r, ~ p r o p e r t i e s ~ o f ~ t h e ~ s e p a r a b l e ~ s u b a l g e b r a s ~ o f ~ a ~}$ G-Galois $\mathrm{H}$-separable extension $\mathrm{S}$ of $\mathrm{S}^{\mathrm{G}}$ are given when $\mathrm{S}^{\mathrm{G}}$ is a projective separable $\mathrm{C}^{\mathrm{G}}$-algebra.
\end{abstract}

KEY WORDS AND PHRASES: Azumaya algebras, Galois extensions, H-separable extensions, Skew group rings.

1991 AMS SUBJECT CLASSIFICATION CODES: 16S30; 16 W20.

\section{INTRODUCTION}

DeMeyer [1] and Kanzaki [2] studied central Galois algebras and Galois extensions whose center is a Galois algebra with Galois group induced by and isomorphic with the group of the extension. These two types of Galois extensions were recently generalized to a bigger class of Galois Azumaya extensions [3]

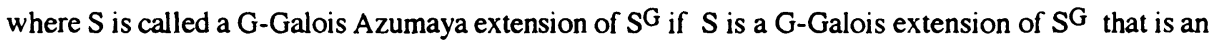
Azumaya $C^{G-a l g e b r a ~ w h e r e ~} C$ is the center of $S$ and $S^{G}$ is the subring of elements fixed under each element of G. Sugano [4] investigated a G-Galois H-separable extension of $S^{G}$, and recently, Szeto [5] proved that a $\mathrm{G}$-Galois $\mathrm{H}$-separable extension $S$ of $\mathrm{S}^{\mathrm{G}}$ that is a projective separable $\mathrm{C}^{\mathrm{G}}$-algebra if and only If $\mathrm{S}$ is a $\mathrm{C}^{\mathrm{G}}$-Azumaya algebra. We call such an $\mathrm{S}$ a GHS-extension. It will be shown that the skew group ring $S * G$ is a $G^{\prime} H S$-extension if and only if $S$ is a $G$-Galois extension of $S^{G}$ that is a projective separable $C^{G}$-algebra, where $G^{\prime}$ is the inner automorphism group of $S * G$ induced by $G$. Moreover, properties of some separable subalgebras of a GHS-extension are also given.

\section{PRELIMINARIES}

Throughout, $S$ is a ring with $1, G$ a finite automorphism group of $S$ of order $n$ invertible in $S, C$ the center of $S$, and $S^{G}$ the subring of elements fixed under each element in $G$. $S$ is called a separable extension of a subring $T$ if there exist $\left\{a_{i}, b_{i}\right.$ in $\left.S / i=1,2, \ldots, m\right\}$ for some integer $m$ such that $\sum a_{i} b_{i}=1$ and $\sum s a_{i} \otimes b_{i}=\sum a_{i} \otimes b_{i} s$ for each $s$ in $S$ where $\otimes$ is over $T$. We call $\left\{a_{i}, b_{1}\right\}$ a separable system for $S$. $S$ is called an $\mathrm{H}$-separable extension of $\mathrm{T}$ if $\mathrm{S} \otimes_{\mathrm{T}} \mathrm{S}$ is isomorphic with a direct summand of a finite direct sum of $\mathrm{S}$ as a bimodule over $\mathrm{S}$. It is known that an $\mathrm{H}$-separable extension is a separable extension and an Azumaya algebra is an $\mathrm{H}$-separable extension. $\mathrm{S}$ is called a $\mathrm{G}$-Galois extension of $\mathrm{S}^{\mathrm{G}}$, if there exist $\left\{c_{i}, d_{i} / i=1,2, \ldots, k\right\}$ in $S$ for some integer $k$ such that $\sum c_{i} d_{i}=1$ and $\Sigma c_{1} g\left(d_{i}\right)=0$ for each $g \neq 1$ in $G$.

We call $\otimes\left\{c_{i}, d_{i}\right\}$ a G-Galois system for $S$. 


\section{SKEW GROUP RINGS}

In this section, we shall show that $S * G$ is a G'HS-extension if and only if $S$ is a G-Galois extensionof $S^{G}$ that is a projective separable $C^{G}$-algebra, and give some properties of the separable subalgebras of an G'HS-extension skew group ring.

THEOREM 3.1. By keeping the notations of section $2, S * G$ is a G'HS-extension if and only if $S$ is a G-Galois extension of $S^{G}$ that is a projective separable $C^{G}$-algebra, where $G^{\prime}$ is the inner automorphism group of $\mathrm{S}^{*} \mathrm{G}$ induced by $\mathrm{G}$.

PROOF. Let $S$ be a $G$-Galois extension of $S^{G}$ that is a projective separable $C^{G}$-algebra. Noting that $S$ is a subring of $S * G$, we have that $S * G$ is also a $G^{\prime}-G$ alois extension of $(S * G) G^{\prime}$ with a same Galors system as $S$ where $G^{\prime}$ is the inner automorphism group of $S * G$ induced by $G$ such that the restriction of $G^{\prime}$ to $S_{1 S} G$. Hence $S * G$ is an $H$-separable extension of $(S * G) G^{\prime}([4]$, Corollary 3$)$. Moreover, since $n$ is a unit in $S, S * G$ is a separable extension of $S$. But $S * G$ is a free module over $S$ and $S$ is a $G$-Galois extension of $S^{G}$ that is a projective separable $C^{G}$-algebra by hypothesis, so $S * G$ is a projective separable $\mathrm{CG}^{\mathrm{G}}$-algebra by the transitivity of projective separable extensions. Since the order of $\mathrm{G}^{\prime}$ is $\mathrm{n}$, it is easy to see that $(S * G) G^{\prime}$ is a direct summand of $S * G$ as a two sided $(S * G)^{G^{\prime}}$-module. Noting that $S * G$ is finitely generated and projective module as a right $(S * G) G^{\prime}$-module or a left module, we have that $(S * G) G^{\prime}$ is a projective separable $\mathrm{C}^{\mathrm{G}}$-algebra by the same argument as given in the proof of Lemma 2 in [1]. This completes the sufficiency.

For the necessity, $S * G$ is a projective separable $C^{G}$-algebra by the transitivity of projective separable extensions because $S^{*} G$ is a $G^{\prime}-G$ alois extension of $(S * G) G^{\prime}$ that is a projective separable algebra of $C^{G}$. Hence $S * G$ is an Azumaya algebra of its center $Z$. But $S$ is a free module over $S$ and $n$ is a unit in $S$, so $S$ is a finitely generated and projective left $S *$ G-module by the proof of Proposition 2.3 in [6] where gs = $\mathrm{g}(\mathrm{s})$ for each $\mathrm{s}$ in $\mathrm{S}$ and $\mathrm{g}$ in $\mathrm{G}$. Thus $\mathrm{S}$ is a finitely generated and projective $\mathrm{Z}$-module by the transitivity of finitely generated and projective modules. Noting that 1 is in $C^{G}$ and that $C^{G}$ is contained in $Z$, we have that $S$ is a faithful $Z$-module. Thus $S$ is a progenerator over $Z$. Since $S * G$ is an Azumaya $Z$-algebra, $S$ is a progenerator over $S * G$. Therefore, $S$ is a $G-G a l o i s$ extension of $S^{G}$. Moreover, since $S$ is a direct summand of $S * G$ as a $C{ }^{G}$-module and $S * G$ is a finitely generated and projective $C^{G}$-module, $S$ is also a finitely generated and projective $C^{G}$-module. Now $n$ is a unit in $S$, so $S^{G}$ is a $S^{G}$-direct summand of $S$. This implies that $S^{G}$ is a finitely generated and projective $C^{G}$-module. So, it suffices to show that $S^{G}$ is a separable $C^{G}$-algebra. In fact, since $S$ is a progenerator over $C^{G}$ (for 1 is in $C^{G}$ ), $\operatorname{Hom}_{C G}(S, S)$ is an Azumaya $C^{G}$-algebra. But $S^{G} \approx \operatorname{Hom}_{S * G}(S, S)=$ the commutator of $S * G$ in $\operatorname{Hom}_{C}(S, S)$, so $S^{G}$ is a separable $C^{G}$-algebra (for so is $S * G$ ) by the commutant theorem for Azumaya algebras ([6], Theorem 4.3).

Next we give some properties of the separable subalgebras of $S * G$.

COROLLARY 3.2. If $S^{*} G$ is a G'HS-extension, then, for any subgroup $K$ of $G, S * K$ is a $\mathrm{K}^{\prime}$-Galois extension of $(\mathrm{S} * \mathrm{~K})^{\mathrm{K}^{\prime}}$ that is a separable $\mathrm{C}^{\mathrm{G}}$-algebra where $\mathrm{K}^{\prime}$ is the inner automorphism group of $S^{*} \mathrm{~K}$ induced by $\mathrm{K}$.

PROOF. By Theorem 3.1, $S$ is a G-Galois extension of $S^{G}$ that is a projective $C^{G}$-algebra, so $S$ is a $\mathrm{K}$-Galois extension of $S^{K}$. Hence $S * K$ is a $K^{\prime}-G$ alors of $(S * K)^{K^{\prime}}$. Noting that the order of $K^{\prime}$ is a unit in $\mathrm{S}$, we have that $(\mathrm{S} * \mathrm{~K})^{\mathrm{K}^{\prime}}$ is a direct summand of $\mathrm{S}^{*} \mathrm{~K}$ as a $(\mathrm{S} * \mathrm{~K})^{\mathrm{K}^{\prime}}$-module. But $\mathrm{S}^{*} \mathrm{~K}$ is a projective separable $\mathrm{C}^{\mathrm{G}}$-algebra, so $(\mathrm{S} * \mathrm{~K})^{\mathrm{K}}$ is a separable $\mathrm{CG}^{\mathrm{G}}$-algebra by the same argument as given in the proof of Lemma 2 in [1].

Let $V_{S}(T)$ be the commutator subring of the subring $T$ in $S$, and $Z$ the center of $S * G$. We give an expression of the commutator subring of $(S * G)^{K^{\prime}}$ in $S * G$. 
THEOREM 3.3. If $S * G$ is a $G^{\prime} H S$-extension, then (1) for any subgroup $K$ fo $G, V_{S * G}\left((S * G) K^{\prime}\right)$ $=\mathrm{ZK}$, and (2) $\mathrm{ZK}$ is an Azumaya algebra over its center $\mathrm{D}$ such that $\mathrm{D}=\mathrm{D}^{\mathrm{K}}$.

PROOF. (1) By Theorem 3.1, $S * G$ is a separable $C^{G}$-algebra, so $S * G$ is an Azumaya $Z$-algebra. Since $\mathrm{n}$ is a unit in $\mathrm{S}$, the order of $\mathrm{K}$ is a unit in $\mathrm{S}$; and so $\mathrm{ZK}$ is a separable $\mathrm{Z}$-algebra contained in $\mathrm{S} * \mathrm{G}$. Noting that $V_{S * G}(Z K)=(S * G)^{K^{\prime}}$, we have that $(S * G)^{K^{\prime}}$ is a separable $Z$-subalgebra of $S * G$ such that $Z K$ $=V_{S * G}\left((S * G)^{\left.K^{\prime}\right)}\right.$ by the commutant theorem for Azumaya algebras ([6], Theorem 4.3).

(2) Since $S * G$ is a separable $C^{G}$-algebra, $Z$ is a separable $C^{G}$-algebra. Hence $Z K$ is a separable $\mathrm{C}^{\mathrm{G}}$-algebra (for the order of $\mathrm{K}$ is a unit in $\mathrm{Z}$ ). Thus $\mathrm{ZK}$ is an Azumaya $\mathrm{D}$-algebra. It remains to show that $\mathrm{D}=\mathrm{D}^{\mathrm{K}^{\prime}}$. Clearly, $\mathrm{D}^{\mathrm{K}^{\prime}} \subset \mathrm{D}$. Conversely, let $\mathrm{d}$ be an element in $\mathrm{D}$. Then $\mathrm{gd}=\mathrm{dg}$ for each $\mathrm{g}$ in $\mathrm{K}$, so $\mathrm{gdg}^{-1}=\mathrm{d}$ for each $\mathrm{g}$ in $\mathrm{K}$. Hence $\mathrm{d}$ is in $\mathrm{DK}^{\mathrm{K}}$.

The follwoing consequences are immediate.

COROLLARY 3.4. Let $S^{*} G$ be an $G^{\prime} H S-e x t e n s i o n$. If $K$ is an abelian subgroup of $G$, then $(\mathrm{S} * \mathrm{G})^{\mathrm{K}^{\prime}}$ is an Azumaya $\mathrm{ZK}$-algebra.

PROOF. By the proof of Corollary 3.3, $(\mathrm{S} * \mathrm{G})^{\mathrm{K}^{\prime}}$ and $\mathrm{ZK}$ are separable subalgebras of the Azumaya $\mathrm{Z}$-algebra $\mathrm{S} * \mathrm{G}$ such that $\mathrm{V}_{S * \mathrm{G}}(\mathrm{ZK})=(\mathrm{S} * \mathrm{G})^{\mathrm{K}^{\prime}}$ and $\mathrm{V}_{\mathrm{S} * \mathrm{G}}\left((\mathrm{S} * \mathrm{G})^{\mathrm{K}}\right)=\mathrm{ZK}$, so $\mathrm{ZK}$ is contained in the center of $(S * G)^{K^{\prime}}$ and the center of $(S * G)^{K^{\prime}}$ is contained in $Z K$. Thus $Z K$ is the center of $(S * G)^{K^{\prime}}$.

COROLLARY 3.5. Let $S * G$ be a G'HS-extension. Then (1) if $(S * G)^{K^{\prime}}$ is a commutative ring,

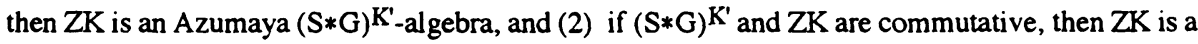
splitting ring for the Azumaya $Z$-algebra $S * G$.

PROOF. (1) It is immediate by the same argument of Corollary 3.4-(1). (2) Since $(S * G)^{K^{\prime}}$ and $Z K$ are separable subalgebras of the Azumaya $Z$-algebra $S * G$ such that $V_{S * G}\left((S * G) K^{\prime}\right)=Z K$ and $\mathrm{V}_{\mathrm{S} * \mathrm{G}}(\mathrm{ZK})=(\mathrm{S} * \mathrm{G})^{\mathrm{K}^{\prime}},(\mathrm{S} * \mathrm{G})^{\mathrm{K}^{\prime}}=\mathrm{ZK}$ (for $(\mathrm{S} * \mathrm{G})^{\mathrm{K}^{\prime}}$ and $\mathrm{ZK}$ are commutative) such that $\mathrm{V}_{\mathrm{S} * \mathrm{G}}(\mathrm{ZK})=\mathrm{ZK}$. Hence $Z K$ is a maximal commutative separable subalgebra of $S * G$. Thus $Z K$ is a splitting ring for the Azumaya algebra $S * G([6]$, Theorem 5.5).

\section{SEPARABLE ALGEBRAS}

In this section, we shall give a property of a separable subalgebra of any GHS-extension similar to Theorem 3.3. Let $T$ be a subalgebra of $S$ over $C G$. The commutator subring of $T$ in $S$ is denoted by $T^{\prime}$.

THEOREM 4.1. Let $S$ be a GHS-extension and $T$ a separable $C^{G}$-subalgebra of $S$, and $\mathrm{K}=\{\mathrm{g}$ in $\mathrm{G} / \mathrm{g}(\mathrm{t})=\mathrm{t}$ for each $\mathrm{t}$ in $\mathrm{T}\}$. Then $\mathrm{T}^{\prime}$ is invariant under $\mathrm{K}$ and an Azumaya $\mathrm{DK}^{\mathrm{K}}$-algebra, where $D$ is the center of $T^{\prime}$ and $K^{\prime \prime}$ is the restriction of $K$ to $T^{\prime}$.

PROOF. Since $t t^{\prime}=t^{\prime} t$ for each $t$ in $T$ and $t^{\prime}$ in $T^{\prime}, \operatorname{tg}\left(t^{\prime}\right)=g\left(t^{\prime}\right) t$ for each $g$ in $K$. Hence $T^{\prime}$ is invariant under $\mathrm{K}$.

Next, since $S$ is a GHS-extension, $V_{S}\left(V_{S}\left(S^{G}\right)\right)=S^{G}\left([4]\right.$, Proposition 4-1). Hence $C=V_{S}(S) \subset$ $V_{S}\left(V_{S}\left(S^{G}\right)\right)=S^{G}$. This implies $C=C^{G}$. Noting that $S$ is a separable $C^{G}$-algebra (for $S$ is a GHSextension), we have that $S$ is an Azumaya $C^{G}$-algebra. But $T$ is a separable subalgebra of $S$, so $T^{\prime}$ is also a separable subalgebra of $S$ such that $V_{S}\left(T^{\prime}\right)=T$ by the commutant theorem for Azumaya algebras ([6], Theorem 4.3). Let $D$ be the center of $T^{\prime}$. Then $D \subset V_{S}\left(T^{\prime}\right)=T \subset S^{K}$. Thus $D=D^{K}$ " where $K^{\prime \prime}$ is the restriction of $\mathrm{K}$ to $\mathrm{T}^{\prime}$. The proof is complete.

COROLLARY 4.2. Let $\mathrm{S}$ be a GHS-extension, $\mathrm{T}$ a separable $\mathrm{C}^{\mathrm{G}}$-algebra of $\mathrm{S}$, and $\mathrm{N}=$

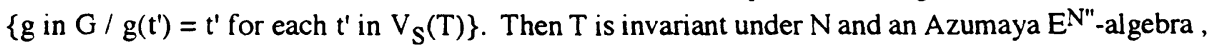
where $E$ is the center of $T$ and $N^{\prime \prime}$ is the restriction of $N$ to $T$.

PROOF. By the proof of Theorem 4.1, $\mathrm{T}$ and $\mathrm{T}^{\prime}\left(=\mathrm{V}_{\mathrm{S}}(\mathrm{T})\right)$ are separable subalgebras of the Azumaya $C^{G}$-algebra $S$ such that $T=V_{S}\left(T^{\prime}\right)$, so the corollary is immediate by Theorem 4.1.

By Theorem 3.1 in [5], Corollary 4.2 implies the following consequence.

COROLLARY 4.3. By keeping the notations and hypotheses of Corollary 4.2, $\mathrm{T}$ is a N"HS-extension. 
We conclude the paper with two examples: (1) $S$ is a G-Galois extension of $S^{G}$ that is a projective separable $C^{G}$-algebra, but not an $\mathrm{H}$-separable extension of $S^{G}$, and (2) $S$ is a GHS-extension.

Example 1. Let $\mathrm{Q}$ be the rational field, $\mathrm{Q}[\sqrt{2}]$ the $\mathrm{G}-\mathrm{Galois}$ extension of $\mathrm{Q}$ with $\mathrm{G}$ alois group $G=\{1, g\}$ where $g(\sqrt{2})=-\sqrt{2}$ and $S=M_{2}(Q[\sqrt{2}])$, the matrix ring of order 2 over $Q[\sqrt{2}]$.

Let $G^{\prime}=\left\{1, g^{\prime}\right\}$ where $g^{\prime}\left(\left[a_{i j}\right]\right)=\left[g\left(a_{i j}\right]\right.$ for all $\left[a_{i j}\right]$ in $S$. Then

(1) $S$ is a $G^{\prime}-G a l o r s$ extension of $S^{G^{\prime}}$,

(2) $S G^{\prime}=M_{2}(Q)$, the matrix ring of order 2 over $Q$,

(3) $S G^{\prime}$ is a projective separable $Q$-algebra,

(4) the center $C$ of $S$ is $Q[\sqrt{2}]$ and $C^{G}=Q$, and

(5) $\mathrm{S}$ is not an $\mathrm{H}$-separable extension of $\mathrm{S}^{\mathrm{G}^{\prime}}$ because $\mathrm{C} \neq \mathrm{C}^{\mathrm{G}}$.

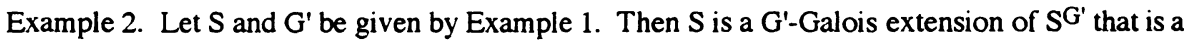
projective separable $C^{G}$-algebra by properties (1) through (4) in Example 1. Then the skew group ring $\mathrm{S}^{*} \mathrm{G}^{\prime}$ is a $\mathrm{G}^{\prime} \mathrm{HS}$-extension by Theorem 3.1 .

ACKNOWLEDGMENT. This work was done under the support of a Caterpillar Fellowship at Bradley University. The paper is revised under the suggestions of the referee. The author would like to thank him for his valuable suggestions.

\section{REFERENCES}

[1] DEMEYER, F. R., Some Notes On The General Galois Theory Of Rings, Osaka J. Math. 2 (1965), $117-127$

[2] KANZAKI, T., On Galois Algebra Over A Commutative Ring, Osaka J. Math. 2 (1965), 309-317.

[3] ALFARO, R. and SZETO, G., Skew Group Rings Which Are Azumaya, Comm. In Algebra 23 (6), (1995), 2255-2261.

[4] SUGANO, K., On A Special Type Of Galois Extensions, Hokkaido J. Math. 9 (1980), 123-128.

[5] SZETO, G. , On A Class Of Azumaya Algebras, Math. Japon. (to appear).

[6] DEMEYER, F. R. and INGRAHAM, E., Separable Algebras Over Commutatıve Rings 181 , Springer-Verlag, Berlin,1971.

[7] IKEHATA, S., Note On Azumaya Algebras And H-Separable Extensions, Math. J. Okayama Univ. 23 (1981), 17-18.

[8] OKAMOTO, H., On Projective H-separable Extensions Of Azumaya Algebras, Results In Mathematics 14 (1988), 330-332. 


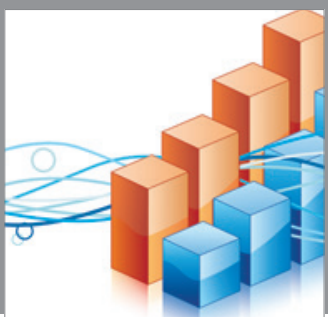

Advances in

Operations Research

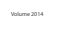

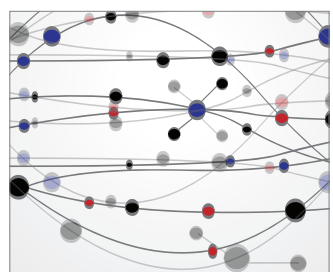

\section{The Scientific} World Journal
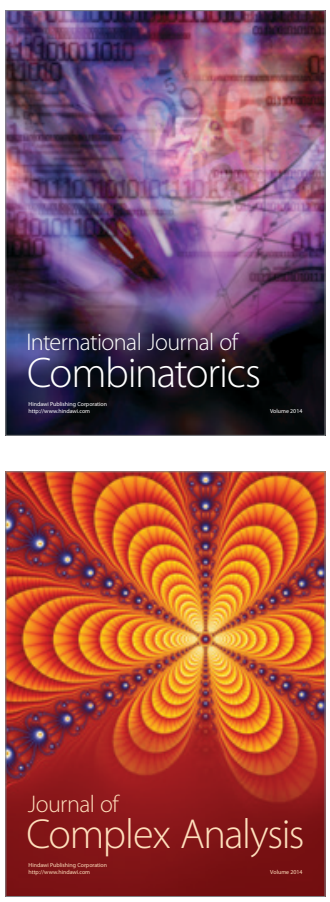

International Journal of

Mathematics and

Mathematical

Sciences
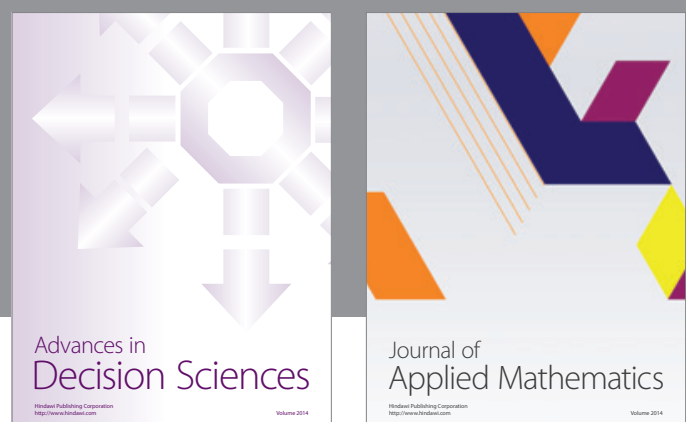

Journal of

Applied Mathematics
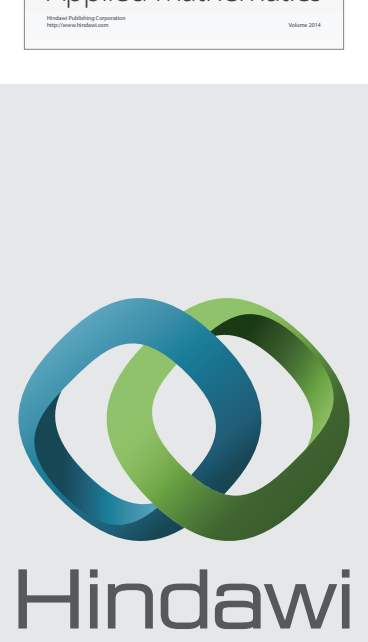

Submit your manuscripts at http://www.hindawi.com
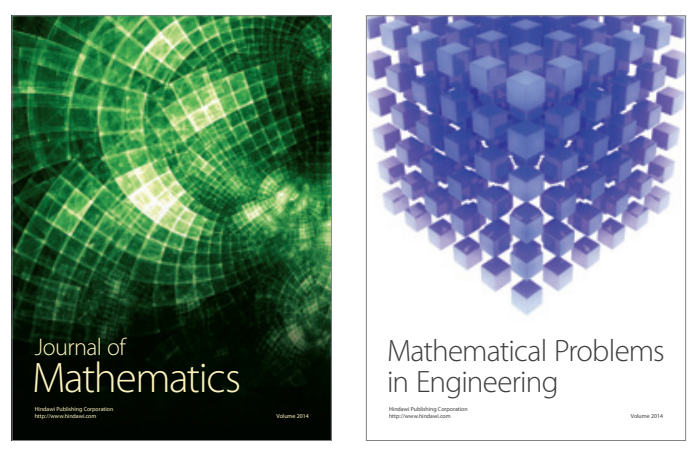

Mathematical Problems in Engineering
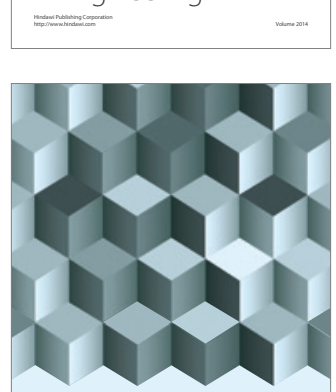

Journal of

Function Spaces
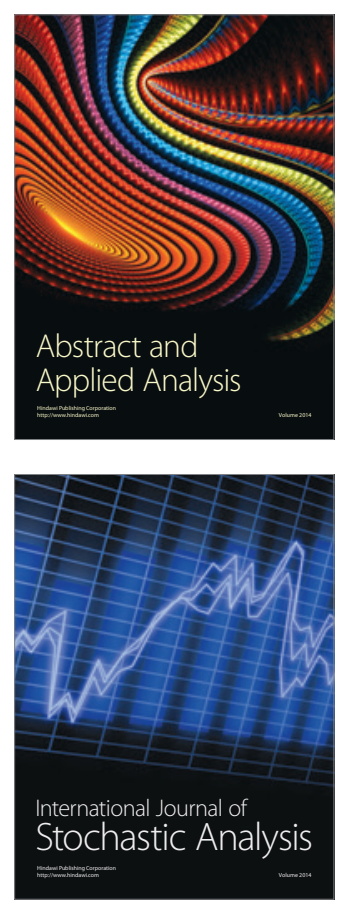

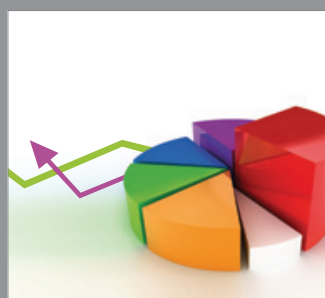

ournal of

Probability and Statistics

Promensencen
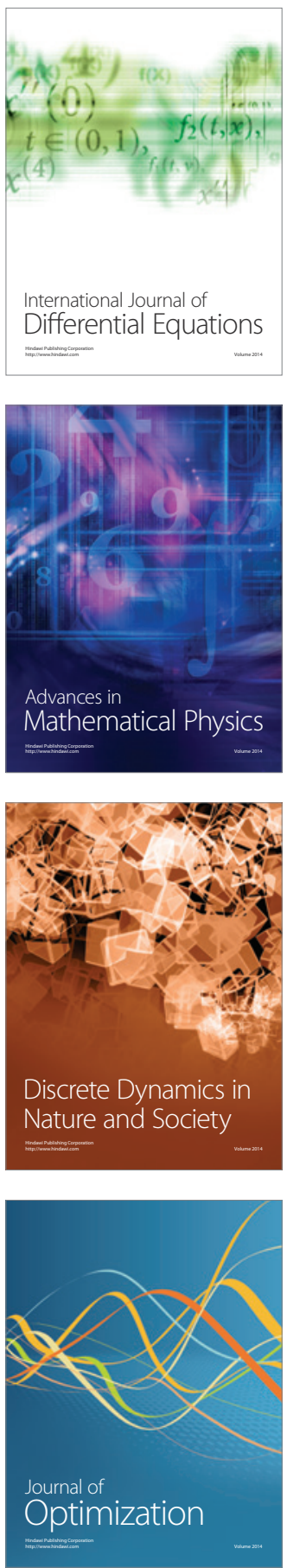